\title{
Esophageal Variceal Bleeding as the First Manifestation of COVID-19 Infection: A Case Report
}

\author{
Christos Sotiropoulos " ${ }^{*}$, MD, MSc, Eftichia Sakka ${ }^{2}$, MD, Georgios Theocharis ${ }^{3}$, MD, PhD, \\ Konstantinos Thomopoulos ${ }^{4}$, MD, PhD,
}

${ }^{1}$ Department of Gastroenterology-Hepatology, University General Hospital of Patras "Holy Mary the Help", Patra, Greece; ORCID: 0000-0001-9233-8700; cr.sotiropoulos@hotmail.com

${ }^{2}$ Department of Internal Medicine, General Hospital of Patras "St Andrew", Patra, Greece; efichem1992@ @otmail.gr ${ }^{3}$ Department of Gastroenterology-Hepatology, University General Hospital of Patras "Holy Mary the Help", Patra, Greece; georgiostheocharis@hotmail.com

${ }^{4}$ Professor and Head Physician of Department of Gastroenterology-Hepatology, University General Hospital of Patras "Holy Mary the Help", Patra, Greece; kxthomo@hotmail.com

*Corresponding author: Christos Sotiropoulos, MD, MSc; cr.sotiropoulos@ hotmail.com

Received 12 December 2021;

Accepted 29 December 2021;

Published 05 January 2022

\begin{abstract}
Liver cirrhosis is a defined liver disease with a wide range of clinical manifestations. Variceal bleeding is the main source of gastrointestinal hemorrhage among cirrhotic patients induced by several factors, such as alcohol consumption or infections. This is a report of a cirrhotic patient presenting with esophageal variceal bleeding in the context of COVID-19 infection. We report the case of a 53-year-old patient with liver cirrhosis and multifocal hepatocellular carcinoma presenting with upper gastrointestinal bleeding as the first manifestation of COVID-19 infection. Upon admission, the patient had no symptoms suggestive of a respiratory tract infection or any contact with positive SARS-CoV-2 individual and upper gastrointestinal endoscopy revealed variceal hemorrhage. After a few hours the patient manifested with fever, cough and dyspnea and a SARS-CoV-2 polymerase chain reaction test obtained was positive. The patient was initially treated with endoscopic band ligation and transferred in the COVID-19 infection clinic, where after a few days of hospitalization he passed away. The devastating pandemic of coronavirus disease 2019 had altered the pathophysiology and clinical presentation of several chronic diseases. This case report suggests that coronavirus disease as a potential triggering factor of variceal bleeding.
\end{abstract}

Kevwords: COVID-19 infection; gastrointestinal hemorrhage; hepatocellular carcinoma; liver cirrhosis; variceal bleeding

\section{Introduction}

Liver cirrhosis is traditionally dichotomised in compensated and decompensated form and the transition to decompensated state is defined when one of the following hallmarks occurs: ascites, variceal bleeding, hepatorenal syndrome or hepatic encephalopathy [1]. Gastrointestinal bleeding is one of the most frequent decompensating events in cirrhotic patients with the majority owing to esophageal varices ${ }^{[2]}$. In a hospitalized patient the main aim is to control the hemorrhage and prevent re-bleeding events, while a thorough investigation is conducted in order to detect the exact triggering factor of decompensation ${ }^{[2]}$. In a hemodynamically stable patient upper endoscopy and treatment with endoscopic band ligation is recomended as the standard of care ${ }^{[2]}$. We present a case of a cirrhotic patient with esophageal variceal bleeding induced by COVID-19 infection.

\section{Case Presentation}

A male 53-year-old patient with known liver cirrhosis and multifocal hepatocellular carcinoma (HCC) (Figure 1), in the setting of chronic ethylation and hepatitis $\mathrm{C}$, was admitted to the hospital's emergency department due to hematemesis and black, tarry stools. Upon admission the patient dad no other symptoms suggestive of a COVID-19 infection, such as cough, sore throat, shortness of breath, anosmia, ageusia, rhinorrhea, headache or other gastrointestinal symptoms and denied any contact with positive SARS-CoV-2 individual. 


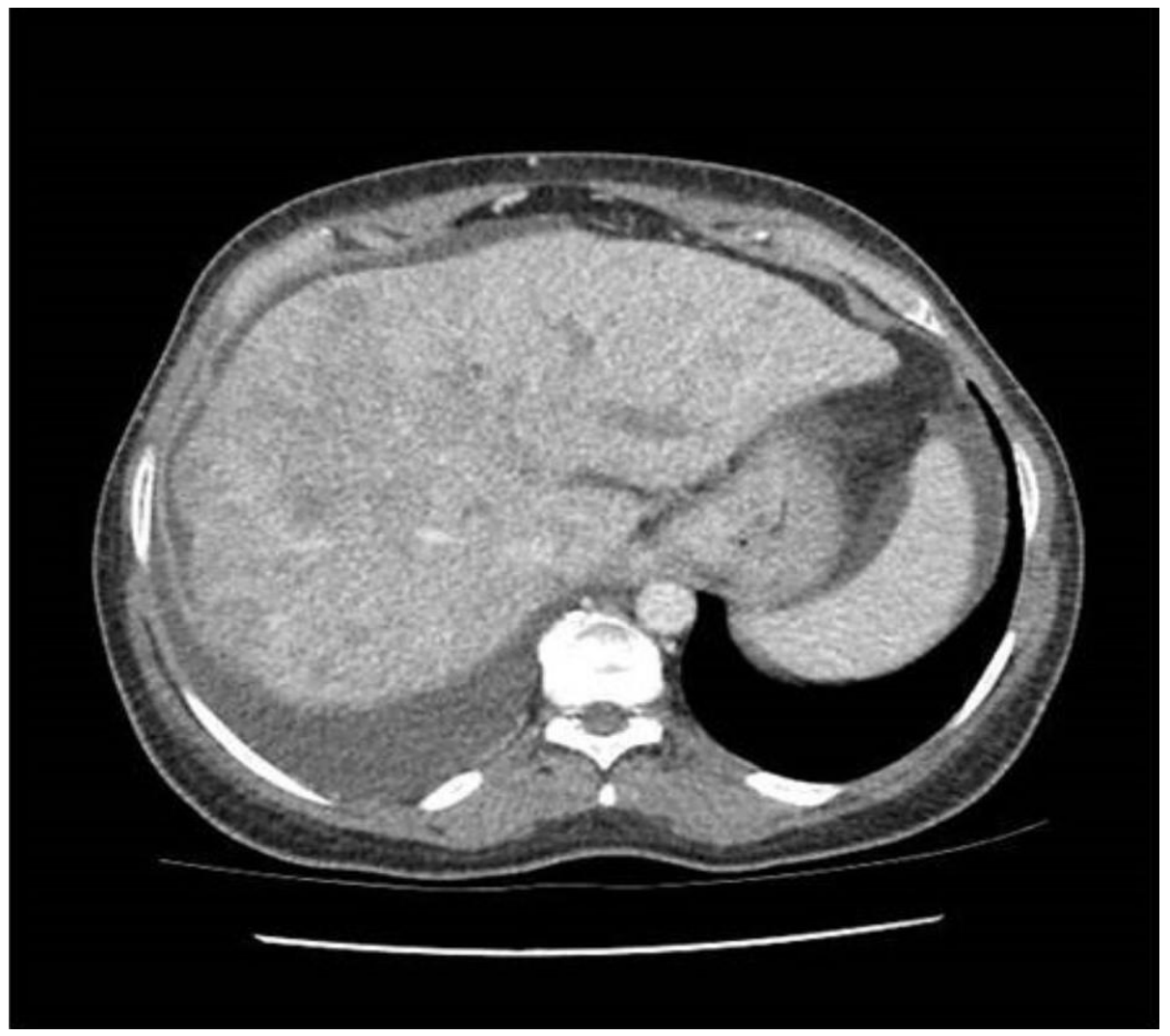

Figure 1: Computed Tomography scan of multifocal hepatocellular carcinoma.

The patient had a recent hospitalization, one month ago, for portal vein thrombosis treated with low molecular weight heparin (LMWH). The patient on the examination was hemodynamically stable without electrocardiographic lesions indicative of ischemia. The findings from the physical examination included moderate ascites, no signs of encephalopathy and reduced respiratory whispering of the right middle and lower pulmonary fields.

The laboratory values revealed macrocytic anemia (Hct: 28,00\%, Hb: 9,40 g/dl, MCV: 104,10 fl, MCH: 34,90 pg), impaired coagulation mechanism (INR: 1,03, aPTT:43,9 sec), abnormal liver function tests (TBL: 8,55 mg/dl, DBL: $5,60 \mathrm{mg} / \mathrm{dl}$, AST: $204 \mathrm{U} / \mathrm{l}$, ALT: $74 \mathrm{U} / 1, \gamma$-GT: $309 \mathrm{U} / \mathrm{l}$, ALP: $414 \mathrm{U} / \mathrm{l}$, ALB: 3,0 gr/dl) and a Child-Pugh Class $\mathrm{C}$ patient. Abdominal paracentesis was not suggestive for spontaneous bacterial peritonitis and the blood and urine cultures obtained were negative. The chest X-ray examination revealed patchy airspace opacification and pleural effusion on the right side (Figure 2).

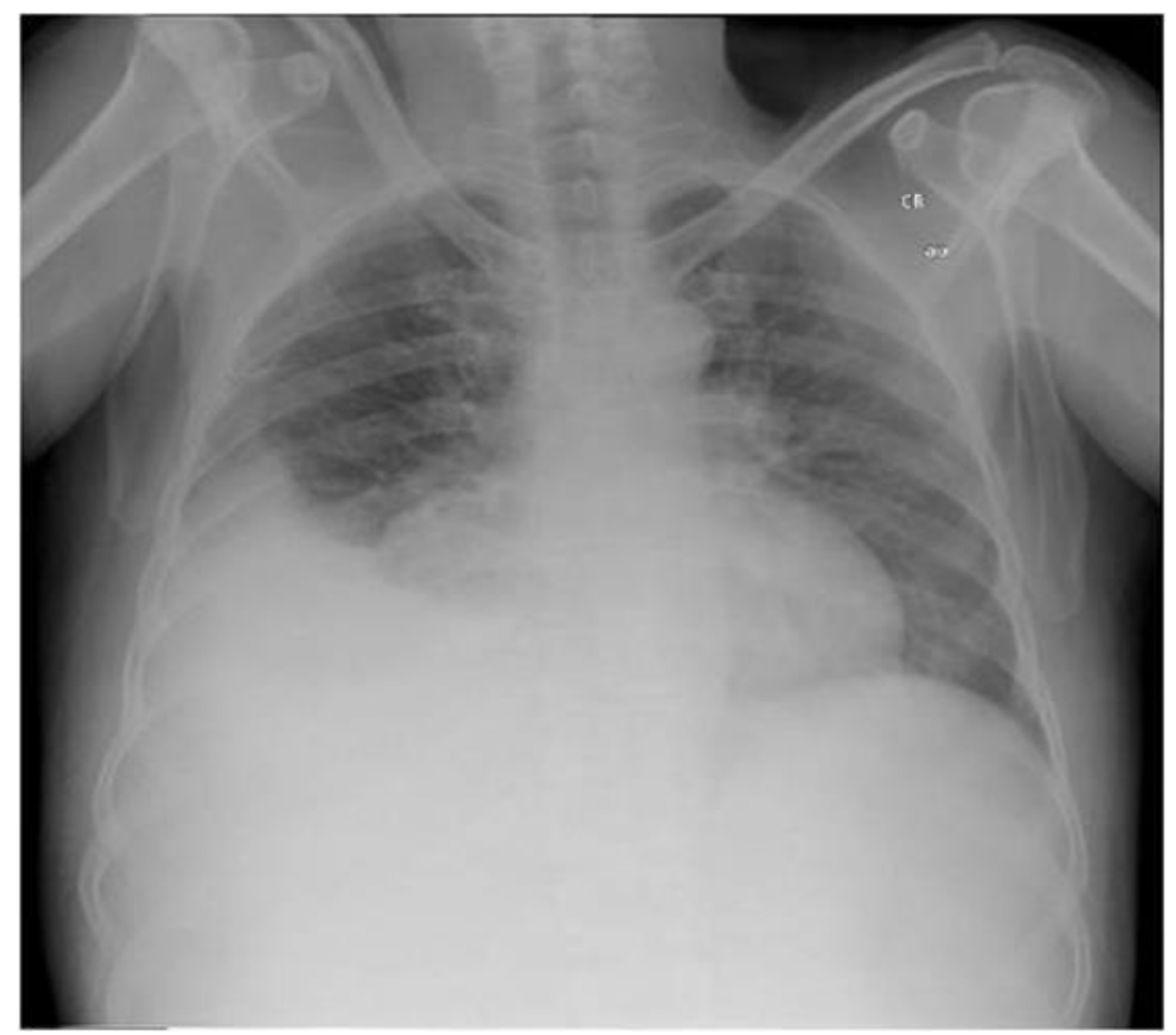

Figure 2: Chest X-ray examination showing patchy airspace opacification and pleural effusion on the right side. 
On the admission, the pathological chest X-ray, the current COVID-19 pandemic and a possible underlying infection as the cause of variceal bleeding led us to suspect the COVID-19 infection as a triggering factor. A rapid antigen test for SARSCOV 2 was negative and then a PCR test for SARS-COV 2 was
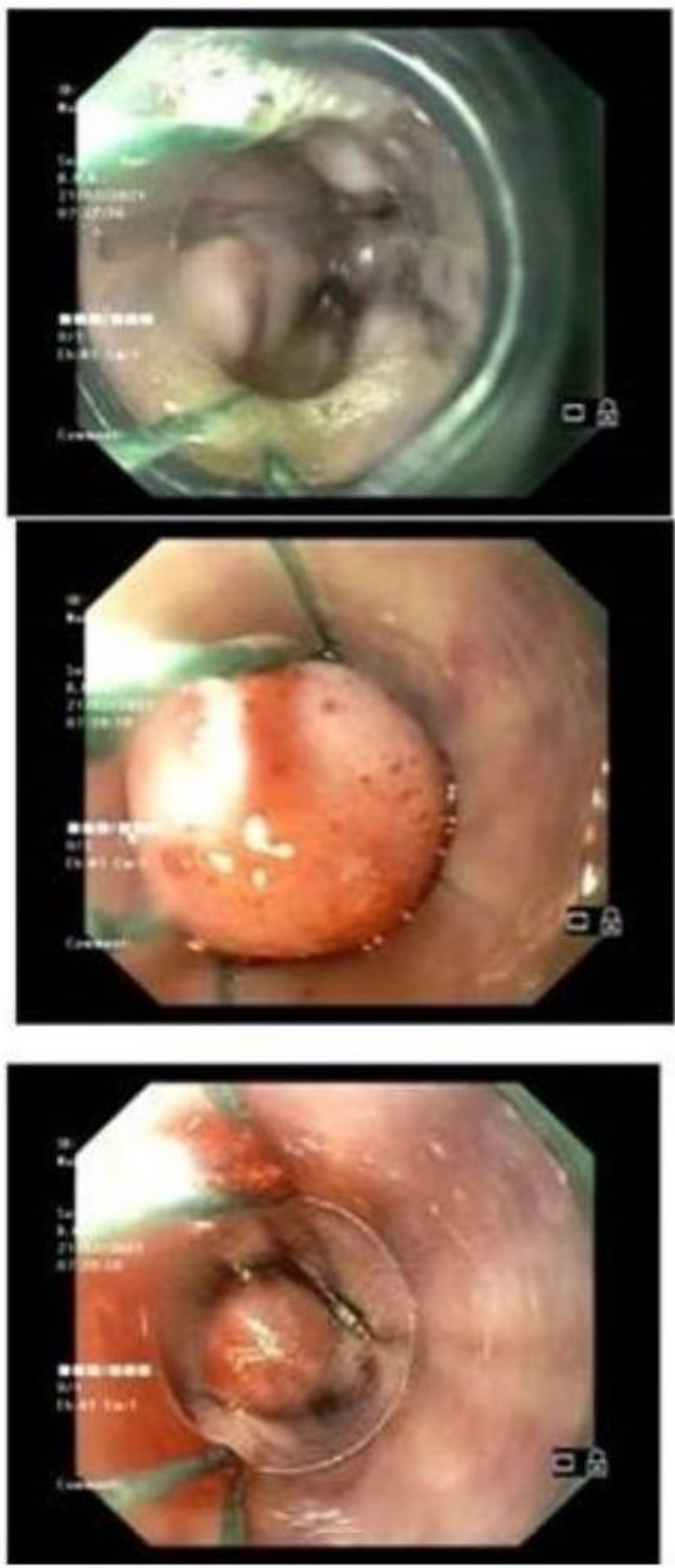

Figure 3: Endoscopic band ligation of grade II-III esophageal varices with red spots.
After a few hours of the admission, the patient presented fever (up to $38^{\circ} \mathrm{C}$ ) with concomitant paroxysmal non-productive cough and dyspnea. Polymerase chain reaction (PCR) COVID-19 testing was positive and the patient was transferred to the COVID-19 infection clinic. Except for complete blood count and liver function tests, further lab workup included elevated D-dimers $(2.8 \mu \mathrm{g} / \mathrm{ml})$, abnormal lactate dehydrogenase (489 U/l), normal creatine kinase, normal high-sensitive cardiac troponin and C-reactive protein and highly elevated ferritin $(3.020 \mathrm{ng} / \mathrm{ml})$. The patient was treated with intravenous corticosteroids and oxygen therapy, without anticoagulants (due to bleeding event). After a few days of obtained. The patient was immediately administered an intravenous somatostatin solution and within 12 hours of admission underwent gastroscopy which revealed grade II-III esophageal varices with red spots, where endoscopic band ligation was performed (Figure 3).
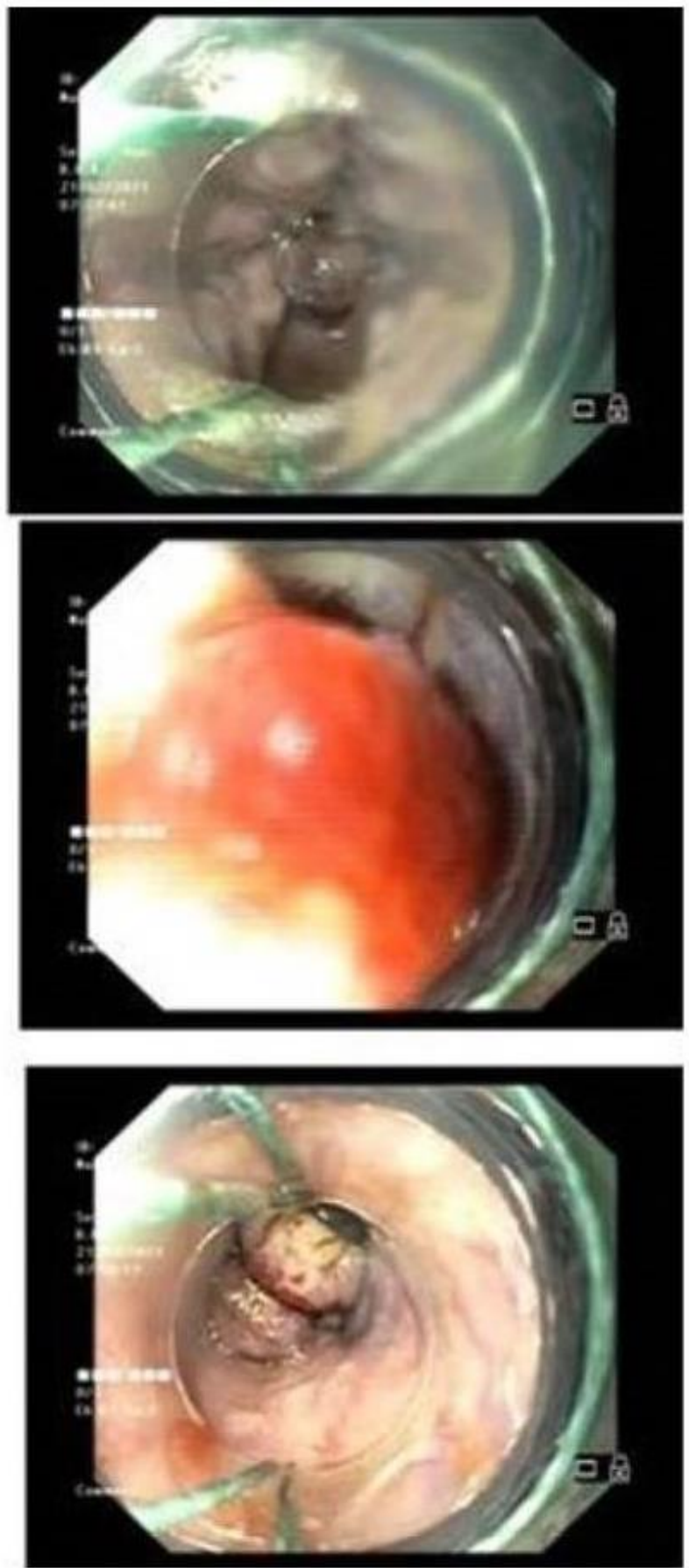

hospitalization he passed away due to decompensated cirrhosis in the context of COVID-19 infection.

To our knowledge, this is the first case report of a patient opening a COVID-19 state with esophagus variceal hemorrhage.

\section{Discussion}

At the time of speaking, COVID-19 infection has rapidly spread worldwide ${ }^{[3]}$ and the clinical manifestations and the disease course in patients with decompensated cirrhosis and COVID-19 infection are incompletely studied ${ }^{[4]}$. Apart from the great thrombotic risk in COVID-19 patients, the "cytokine storm", a massive systemic 
inflammation induced by the COVID-19 infection, has the potential to promote bleeding events ${ }^{[3]}$. The aim of this case report is to monitor COVID-19 infection as a possible risk factor that triggers variceal hemorrhage in patients with liver cirrhosis ${ }^{[5]}$.

Severity of the liver disease is a well-established risk factor of variceal hemorhage in cirrhotic patients and has strong association with prognosis ${ }^{[6]}$. Esophageal variceal bleeding is a fatal complication of cirrhosis and is associated with high mortality ${ }^{[6]}$ and prevention of variceal rupture is a crucial part in the management of cirrhotic patients ${ }^{[1]}$. The most common precipitating events are active alcoholism, bacterial infections and sepsis and relapse of chronic hepatitis B [1,7]. Less common factors are acute hepatitis $\mathrm{A}$ or hepatitis $\mathrm{E}$ virus infection ${ }^{[7]}$. Last but not least, HCC is considered one of the most significant factors that have been associated with variceal bleeding ${ }^{[6]}$.

Given that variceal hemorrhage is one of the leading causes of death in cirrhotic patients, understanding how COVID-19 infection influences the clinical outcome in this setting is crucial

${ }^{[4]}$. This is an effort to better understand the COVID-19 infection as a possible contributing factor to esophageal variceal bleeding ${ }^{[4]}$. Our data suggest that COVID-19 infection might be a causing and/or a contributing factor for variceal hemorrhage [4] and up to our knowledge, this is the first report describing a patient with variceal bleeding induced by COVID-19 infection ${ }^{[8]}$.

\section{Conclusions}

The rapidly expanding coronavirus disease 2019 had a major impact on several chronic diseases both in terms of pathophysiological mechanisms and clinical outcomes. The current case report suggests that coronavirus disease 2019 is a potential triggering factor of variceal bleeding. Nevertheless, it is recognisable that our single case cannot replace larger series of cases to confirm our hypothesis, so further studies will shed light on the association of COVID-19 infection and variceal bleeding.

\section{Abbreviations}

COVID-19, coronavirus disease 2019; HCC, hepatocellular carcinoma; LMWH, low molecular weight heparin; Hct, hematocrit; $\mathrm{Hb}$, hemoglobin; $\mathrm{MCV}$, mean corpuscular volume; $\mathrm{MCH}$, mean corpuscular hemoglobin; INR, international normalized ratio; aPTT, activated partial thromboplastin time; TBL, total bilirubin; DBL, direct bilirubin; AST, aspartate aminotransferase; ALT, alanine transaminase; $\gamma$-GT, gammaglutamyl transferase; ALP, alkaline phosphatase; ALB, albumin; PCR, polymerase chain reaction.

\section{Declarations}

\section{Ethics approval and consent to participate}

All procedures performed in this case report were in accordance with all the ethical standards and an informed consent was obtained from the patient included in this case report. This study did not violate any national or international laws on human, animal and environmental rights.

\section{Funding}

No funding was received for this work.

\section{Conflict of Interest disclosure}

The authors declare that there are not conflicts of interest.

\section{Consent for publication}

An informed consent for publication was given to the patient included in this case report. All authors of this paper have read and approved the final version submitted. We confirm in this statement that written consent to publish this information was obtained from study participants.

\section{Authors' contributions}

CS, GD, GT and KT confirm sole responsibility for the following: study conception and design, data collection, analysis and manuscript preparation. All authors of this manuscript have directly participated in the planning, execution, or analysis of this study and are the only ones responsible for the originality of the scientific content of the manuscript. Finally, all authors have read and approved the manuscript.

\section{Availability of data and material}

The data that support the findings of this case report are available on request from the corresponding author. The data are not publicly available due to restrictions (their containing information that could compromise the privacy of research participants)

\section{Acknowledgments}

Throughout the writing of this case report I have received a great deal of support and assistance. I would first like to thank the coauthors of this case report for valuable assistance in designing, data collection and analysis of this clinical case. I would also like to acknowledge my colleagues from the Department of Gastroenterology-Hepatology in University General Hospital of Patras, for their wonderful collaboration. Finally, authors acknowledge the sacrifices and the great effort of clinicians and healthcare providers all over the world due to COVID-19 pandemic.

\section{References}

[1] Hernaez R, Solà E, Moreau R, Ginès P: Acute-onchronic liver failure: an update. Gut. 2017, 66:541-53. 10.1136/gutjnl-2016-312670

[2] Zanetto A, Garcia-Tsao G: Management of acute variceal hemorrhage. F1000Res. 2019, 8:10.12688/f1000research.18807.1

[3] Schmiderer A, Schwaighofer H, Niederreiter L, Profanter C, Steinle H, Ziachehabi A, Tilg H: Decline in acute upper gastrointestinal bleeding during COVID-19 pandemic after initiation of lockdown in Austria. Endoscopy. 2020, 52:1036-8. 10.1055/a-1178-4656

[4] Qi X, Wang J, Li X, et al.: Clinical course of COVID-19 in patients with pre-existing decompensated cirrhosis: initial report from China. Hepatol Int. 2020, 14:478-82. 10.1007/s12072-020-10051-z

[5] ROMCEA A, TANŢĂU M, SEICEAN A, PASCU O: Variceal bleeding in cirrhotic patients: risk factors, evolution, treatment. Clujul Med. 2013, 86:107-10. 
[6] Kerdsuknirun J, Vilaichone V, Vilaichone RK: Clinical Outcome and Predictive Factors of Variceal Bleeding in Patients with Hepatocellular Carcinoma in Thailand. Asian Pac J Cancer Prev. 2018, 19:3301-5. 10.31557/APJCP.2018.19.11.3301

[7] Arroyo V, Moreau R, Kamath PS, et al.: Acute-onchronic liver failure in cirrhosis. Nat Rev Dis Primers. 2016, 2:16041. 10.1038/nrdp.2016.41

[8] El Kassas M, Al Shafie A, Abdel Hameed AS, Mahdi M: Emergency endoscopic variceal band ligation in a COVID-19 patient presented with hematemesis while on mechanical ventilation. Dig Endosc. 2020, 32:812-5. 10.1111/den.13694
Open Access This article is licensed under a Creative Commons Attribution 4.0 International License, which permits use, sharing, adaptation, distribution and reproduction in any medium or format, as long as you give appropriate credit to the original author(s) and the source, provide a link to the Creative Commons license, and indicate if changes were made. The images or other third party material in this article are included in the article's Creative Commons license, unless indicated otherwise in a credit line to the material. If material is not included in the article's Creative Commons license and your intended use is not permitted by statutory regulation or exceeds the permitted use, you will need to obtain permission directly from the copyright holder. To view a copy of this license, visit https://creativecommons.org/licenses/by/4.0/.

(c) The Author(s) 2021 